\title{
On Extended Normal Distribution Model with Application in Health Care
}

\author{
Bachioua Lahcene*
}

\author{
Department of Basic Sciences, Prep. Year, P.O. Box 2440, University of Hail, Hail, Saudi Arabia
}

\begin{abstract}
In this article, the normal distribution model (NDM) is extended. A introduction to a new Extended Normal Distribution (ENDM) and its derivate models used in many applications is proposed. The author proposes the new model (ENDM) which generalizes the normal distribution models. This class of ENDM approximates an unknown risk-neutral density. The paper discusses different properties of the ENDM. In particular, the applicability of the new model with three parameters in a way to justify the representation of combination of normal distributions is presented. The potential of the proposed distribution for modelling and analyzing statistical data with reference to extensive sets of observations. Statistical properties of the proposed distribution have also been studied. The findings of this work will be useful to practitioners in applied fields of health care.
\end{abstract}

Keywords: Normal Distribution, Goodness-of-fit, Characteristics Function, Survival Function, Mixtures, Health Care.

\section{HISTORY AND DEFINITION}

Normal distribution also called Laplace-gauss distribution, is a probability distribution introduced in mathematical theory of probability by the famous correspondence between Fermat and Pascal in 1654. The studies on normal distribution is in continuation since about 350 years. The origin of normal distribution is credited to French mathematician Abraham de Moivre (1667-1754) in the year 1733, he used the distribution in gambling for determining probabilities [7].

Kruskal \& Stigler used the term normal, apparently Charles S. Peirce (1873) used the term normal in a report of the US Coast Survey. Gauss was the first to propose the normal distribution law, then it was Laplace in 1774 who first proposed the problem of gathering observations which resulted to laplace distribution [13].

Adolphe Quetelet (1796-1874) described the concept of l'homme moyen using the normal distribution with the notion of the bell-shaped curve [22]. De Moivre studied the probability distribution of tossing the coins. He came up with a mathematical expression for finding a probability of 60 toss and frequent tails out of a hundred coin toss.

In 1712, Gravesande tested the hypothesis that male and female births are equally against the actual births in London over the 82 years [10]. Soon after, in 1777, Daniel Bernoulli continued with the contributions

*Address correspondence to this author at the Department of Basic Sciences, Prep. Year, P.O. Box 2440, University of Hail, Hail, Saudi Arabia;

E-mail: bachiouala@hotmail.com of Legendre (1805), Gauss (1809), Laplace (1812), Bessel $(1818,1838)$, Bravais (1846), Airy (1861), and Airy (1861) [23].

In the 1877, Galton used the term "deviated normally" for the first time in a distribution called the law of deviation. However, later he used term "normal" systematically" in Natural Inheritance of the book entitled normal variability. Afterwards, Galton used the term "normal curve" for the normal curve of distributions, but he did not explained the reason for terming it as normal [9].

Carl Friedrich Gauss applied the normal distribution in understanding measurement of errors in fields of geography and astronomy. The contributions of Galton (1875, 1889), Helmert (1876), Tchebyshev (1890), Edgeworth (1883, 1892, 1905), Pearson (1896), Markov (1899, 1900), Lyapunov (1901), Charlier(1905), and Fisher $(1930,1931)$ are of importance [1].

Laplace while deriving the central limit theorem, discovered the normal distribution. Adrain (1808) and Gauss (1809) developed a formula for normal distribution and proved that errors fit considerably with the distribution [11]. The term Laplace curve or normal curve helped in correcting the assumption that all the distribution of frequency is non normal [23]. A clear explanation is evident in the central limit theorem by Laplace [14].

The law of distribution started attracting researchers in west such as C. S. Peirce (1873), Sir Francis Galton (1879) and Wilhelm Lexis (1879) [23]. The work of Karl E. Pearson (1920) influenced many other researchers to work in the field of distribution theories [18]. The 
normal distribution is also referred to as bell curve, and the quantity which is the sum of different and independent processes resulting in normal data. The bell shape curve is common in mostly in statistical reports, survey and quality control and analysis [2].

A detail discussion and introduction to normal distribution and development of the distribution is attributed to Pearson (1967), Patel and Read (1982), Johnson et al. (1994), Stigler (1999) and Wiper et al. (2005) [1]. Normal distribution is defined as value distribution or as a measure of population and represented by the area covered under a normal curve, thus this is the reason the $y$-axis is plotted as probability as a measure of population.

\section{MATHEMATICAL DESCRIPTION AND THEORY}

The normal distribution models is a family of continuous probability distributions which is widely used in statistics and different fields of sciences, having similar form of the graphical representation with same general shape. Assume that $X$ is a random variable, the probability density function of a normal distribution model NGDM (1). The probability density function (pdf) distribution has a single parameter which is given by:

$f_{X}(x, \sigma)=\frac{1}{\sigma \sqrt{2 \pi}} \exp \left\{-\frac{x^{2}}{2 \sigma^{2}}\right\}$

Where $\sigma \succ 0$ represent the shape parameter. On the domain $x \in(-\infty,+\infty)$. Mathematician and Statisticians use the term "Gaussian distribution" for this distribution, in physics and other filed it is termed as "Normal distribution" . Because of the curved flaring shape, social scientists term the distribution as "bell curve".

Feller (1968) uses the symbol $\varphi(x)$ for $P(x)$ in equation (1), but then switches to $N(x)$ in Feller (1971) [ 8]. For the simplicity, call this model by name normal distribution model with one parameter defined by equation (1) and symbolized by the formula NGDM(1). The simplest case of this distribution is known as the standard normal distribution. When $\sigma=1$, the distribution is a pdf given by:

$f_{X}(x)=\frac{1}{\sqrt{2 \pi}} \exp \left\{-\frac{x^{2}}{2}\right\} ;-\infty \prec x \prec+\infty$

It was Pearson who first wrote the distribution in terms of the variance $\sigma^{2}$ as in modern notation. In
1915, fisher added the location parameter $\mu$ to the formula for normal distribution. Other model of normal distribution of one parameter with pdf of distribution has a single location parameter $(\mu)$ form given by [19]:

$f_{X}(x, \mu)=,\frac{1}{\sqrt{2 \pi}} \exp \left\{-\frac{(x-\mu)^{2}}{2}\right\} ;-\infty \prec x \prec+\infty$

The expression of the closed form of the model expressed in equation (1) has been expanded so that a new parameter has been added to become an identifier in terms of two parameters, and the (pdf) form given by [15]:

$f_{X}(x, \mu, \sigma)=\frac{1}{\sigma \sqrt{2 \pi}} \exp \left\{-\frac{(x-\mu)^{2}}{2 \sigma^{2}}\right\} ;-\infty \prec x \prec+\infty$

where $-\infty \prec \mu \prec+\infty$ is the represent the shift in the graph named location parameter and $\sigma \succ 0$ represents the shape parameter. This new form of normal distribution model with two parameters is symbolized by NGDM (2).

The equation in equation (2) is generalized form of equation (1) with $\mu=0$, mean $=\mu$ and variance $=\sigma^{2}$. The generalized Gaussian Normal Distribution (GND) is any of two families of parametric continuous probability distributions on the real number line. Both families includes a shape parameter to the normal distributions.

This version of the generalized gaussian normal distribution has been widely used in modeling. When the concentration of data points around the mean and the behavior of the tail are of importance [5].

The plot of the gaussian normal distribution model with two-parameters represent both the mean $\mu$, and variance $\sigma^{2}$. The graph of its pdf for various values of the parameters $\mu$ and $\sigma$ showed in Figure $1 \mathbf{a} \& \mathbf{b}$.

There are different and many gaussian normal distributions depending on two parameters. The graph of data represent the population with mean $\mu$ and the variance $\sigma^{2}$. Rather than, computations on each new set of parameters for a variety of normal curves, it is quite easy to consider the reference as the "simplest case" of the normal curves, called the standard gaussian normal distribution.

When the value of $\sigma$ is small, pdf results in huge values. The plot of the gaussian normal distribution model its pdf and Cumulative Distribution Function (CDF) for various values of the parameters $\mu$ and $\sigma$ showed in Figure $\mathbf{2 a} \& \mathbf{b}$ ). 


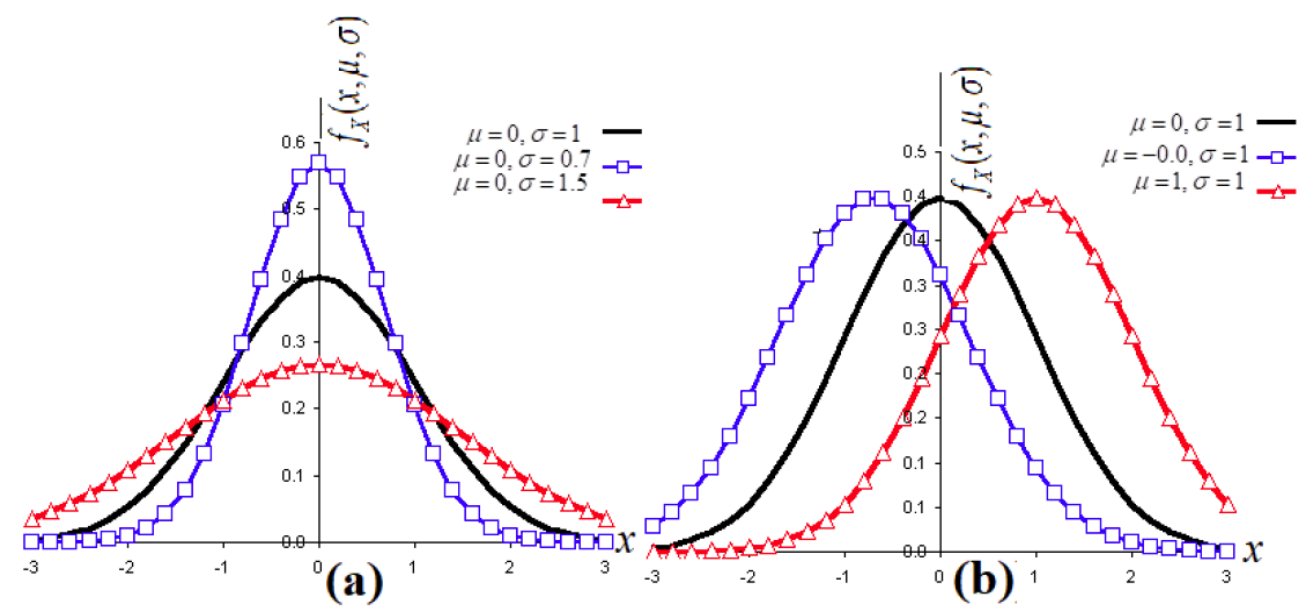

Figure 1: (a \& b): Graph of $f_{X}(x, \mu, \sigma)$ for different values of $\mu$ and $\sigma$.
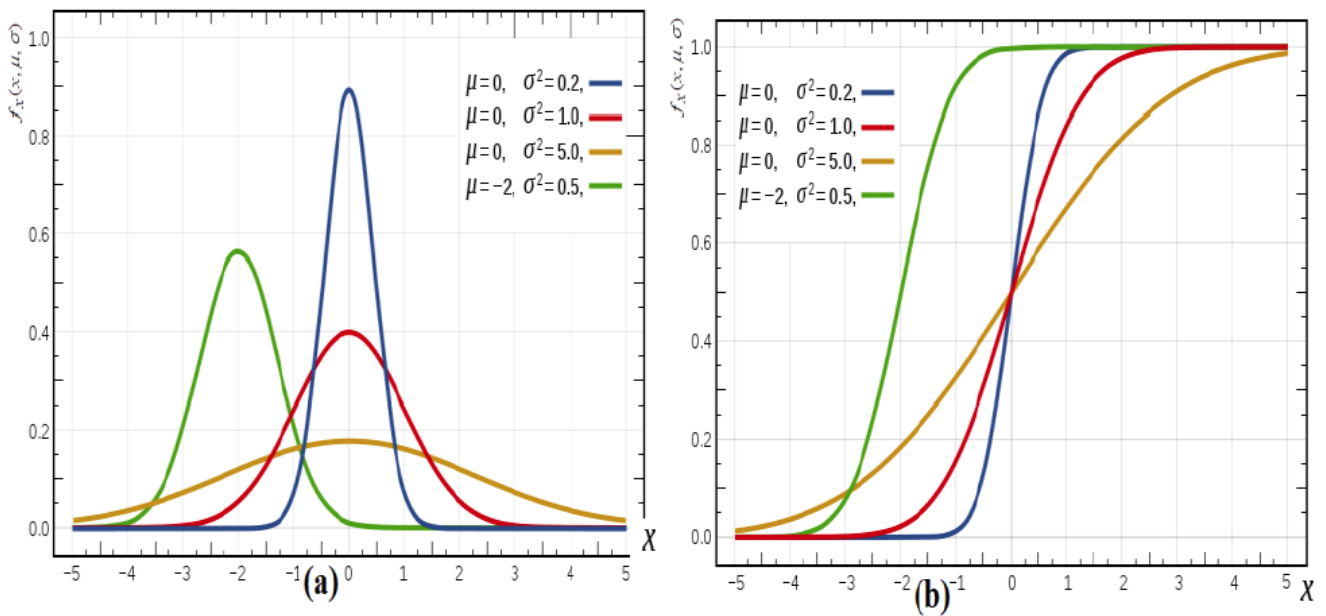

Figure 2: (a \& b): Graph of $f_{X}(x, \mu, \sigma)$ and $F_{X}(x, \mu, \sigma)$ for $\mu$ and $\sigma$ are varied.

In recent years, both kurtosis and skewness were treated with more concern in representing the features of the distribution. This model introduces a new family of skew and symmetric distributions containing the normal family indexed by three parameters.

\section{NEW EXTENDED GAUSSIAN NORMAL DISTRIBUTION}

In the last few 60 years, new classes of distributions were proposed by extending the gaussian normal distribution model. The new model is named as new Extended Gaussian Normal Distribution (EGND). A review of some of these models includes in the exponential family distribution in general form. The pdf of general normal distribution model with three parameters.

In the scope of clarifying some aspects of the extension of the formula of the proposed model we review the specific case, and clarify the development of the threshold parameter formula in the new model presented in terms of pdf as follows:

$$
f_{X}(x, a, b, c)=\exp \left\{a x^{2}+b x+c\right\}=\exp \left\{a\left(x+\frac{b}{2 a}\right)^{2}+\frac{4 a c-b^{2}}{4 a}\right\}
$$

where $a \prec 0$ and we call this new form by name extended general normal distribution with three parameters and symbolized by the formula ENDM(3). The graph of this pdf repressed the parabola opens down with the vertex located at the point.

$\left(-\frac{b}{2 a}, f_{X}\left(-\frac{b}{2 a}, a, b, c\right)\right)=\left(-\frac{b}{2 a}, \frac{4 a c-b^{2}}{4 a}\right)$

Equation (3) can be redrafted in order to be in terms of three parameters representing the mean, the variance and the threshold parameter according to the following new formula: 


$$
f_{X}(x, \lambda, \mu, \beta)=\beta \exp \left\{-\lambda(x-\mu)^{2}\right\}
$$

where $\lambda \succ 0,-\infty \prec \mu \prec+\infty$ and $\beta=\exp \left\{\lambda \mu^{2}\right\} \succ 0$. The parameters of the model expressed during the formulation of equations (3), (4) and the normalized equation for gaussian normal distribution with mean $\mu$, and standard deviation $\sigma$ can be linked in equation (2) in characteristic property (1). Can be fully specified via two parameters: $\mu$ and $\sigma$.

The distribution is denoted by $N(\mu, \sigma)$, It can be shown $\sigma^{2}$ is the variance of $x\left(\sigma^{2}\right.$ is the variance of $\left.x\right)$. There's no rule that says that it should always be better, particularly with something so subjective as image classification in Figure 3.

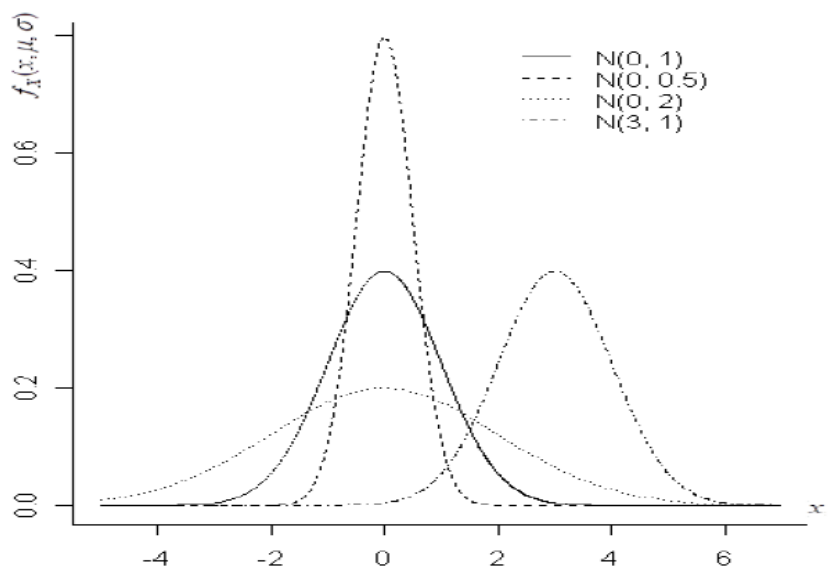

Figure 3: Graph of $f_{X}(x, \mu, \sigma)$ for various values of $\mu$ and $\sigma$.

\section{PROPERTIES OF GAUSSIAN NORMAL DISTRIBUTION MODEL}

The following properties shows the important results of the normal distribution.

Property (1): Every gaussian normal distribution is the exponential of a quadratic function.

Proof: The result follows by illustrated of basic transformation technique methods. Let the function $f_{X}(x)=\exp \left\{a x^{2}+b x+c\right\} \quad$ where $\quad a \prec 0 \quad$ and $c=\frac{b^{2}}{4 a}+\frac{\ln (-a / \pi)}{2}$. In this form, the mean value is $\mu=\frac{-b}{2 a}$ and the standard deviation is $\sigma=\sqrt{(-1 / 2 a)}$.

Property (2): Let $X$ be Random variable with gaussian normal distribution of two parameters NDM (2). Then
1. The area under the curve and over the $x$-axis is unity.

2. It is symmetric to the mean $\mu$ which is at the same time mode $=$ median $=$ mean of the distribution.

3. Its density function has two inflection points is zero and changes sign), located one root of variation away from the mean, namely at $x=\mu-\sigma$ and $x=\mu+\sigma$.

4. It is density function unimodal its first derivative is negative for $x \succ \mu$, zero only at $x=\mu$ and is positive for $x \prec \mu$.

Proof: See the proof of this property represented in reference [17], it is simple and straightforward.

Property (3): Let $x$ be random variable with gaussian normal distribution of two parameters NDM (2).i.e $X \sim N\left(\mu, \sigma^{2}\right)$. Then the standard normal $Z=\frac{X-\mu}{\sigma} \sim N(0,1)$

Proof: By definition of the gaussian normal distribution. The proof of this property is simple and straightforward.

Property (4): If $X_{1}, X_{2}, X_{3}, \ldots, X_{n}$ are independent random variables that are normal distributed with of two parameters NDM (2). Then

1. The total sum of their squares has the chi-square distribution with $n$ degrees of freedom $X_{1}^{2}+X_{2}^{2}+X_{3}^{2}+\ldots . X_{n}^{2} \sim \chi_{n}^{2}$.

2. If $X_{1}, \ldots, X_{n}$ are independent and identically distributed $\mathrm{N}\left(\mu, \sigma^{2}\right)$ where $\mu$ is unknown, then $\frac{(n-1) S^{2}}{\sigma^{2}} \sim \chi_{n-1}^{2}$.

Proof: By definition of the gaussian normal distribution. The proof of this result is simple and straightforward.

Property (5): Let $X$ be Random variable with gaussian normal distribution of two parameters ENDM (2). Then

1. $E(X)=\mu$

2. $\sqrt{V(X)}=\sigma$

3. Skewness $(X)=\operatorname{Kurtosis}(X)=0$ 
Proof: By definition of the normal distribution. Similarly, its proof is simple and straightforward.

Property (6): If $X$ has a gaussian normal distribution $\operatorname{NDM}(2)$ with mean $\mu$ and variance $\sigma^{2}$ then we can analytically evaluate various expectations such as

$E\left(X^{2}\right)=\mu^{2}+\sigma^{2}$

or

$E[\exp (X)]=\exp \left(\mu+\frac{\sigma^{2}}{2}\right)$

Proof: By definition of the gaussian normal distribution. Again, the proof of this result is simple and straightforward.

Property (7): If $X_{1}, \ldots, X_{n}$ are independent and identically distributed $\mathrm{N}\left(\mu, \sigma^{2}\right)$ where $\mu$ is unknown, then $\frac{(n-1) S^{2}}{\sigma^{2}} \sim \chi_{n-1}^{2}$.

Property (8): For an independent random $X_{1}, \ldots, X_{n}$ from a gaussian normal distribution NDM(2) with mean $\mu$ and variance $\sigma^{2}$, the distribution of $\bar{X}$ has these properties:

1. $E[\bar{X}]=\mu$

2. $\operatorname{Var}[\bar{X}]=\frac{\sigma^{2}}{n}$ and $S D[\bar{X}]$ is therefore $\frac{\sigma}{\sqrt{n}}$.

3. If $X_{i} \sim N\left(\mu, \sigma^{2}\right)$, then $\bar{X} \sim N\left(\mu, \frac{\sigma^{2}}{n}\right)$ irrespective of the size of $n$.

\section{Proof}

1. $E[\bar{X}]=E\left[\frac{1}{n} \sum_{1}^{n} X_{i}\right]=\frac{1}{n} \sum_{1}^{n} E\left[X_{i}\right]=\frac{1}{n} n \mu=\mu$.

2. Because we suppose that the random variables are independent, we can also write:

3. $\operatorname{Var}[\bar{X}]=\operatorname{Var}\left[\frac{1}{n} \sum_{1}^{n} X_{i}\right]=\frac{1}{n^{2}} \sum_{1}^{n} \operatorname{Var}\left[X_{i}\right]=\frac{1}{n^{2}} n \sigma^{2}=\frac{\sigma^{2}}{n}$

4. A linear combination of random variables which are normally distributed also has a gaussian normal distribution. The variance and mean are as given above.

\section{MIXTURES OF GAUSSIAN NORMAL DISTRIBUTIONS}

This type of mixture is defined as a variance-mean mixture which is normally distributed. Some extensions of this mixtures have stochastic volatility types and they are constructed via an approach of observation to state space modelling.

Interestingly, NDM (2) distribution is a versatile distribution in its own right and has been utilized in fatigue crack growth and reliability, and health care [20].

We study the mixture model composed of different sub models filtering problem for a partially stochastic process case which are observable in different type of models. The solution of this situation is based on a nonlinear stochastic models with difference equations, which provides a stochastic metallization for parameters of these models with normally distributed observation.

The limited health-based literature reveals several methods, which have been utilized to compute the time of best practice in health care. Early literature used stylized decision problems and simplifying assumptions, such as this model with gaussian normal distribution net benefit, to calculate overall time of best practice analytically via standard statistical tables, but gave no analytic calculation method for partial time of best practice [20].

Extended model with gaussian normal distribution modified with three parameters ENDM (3) is a right skewed distribution and it plays a crucial role in reliability analysis. Due to its edibility and several other interesting properties, it has been a popular alternative to the recent modifications of convergence all families distributions [3].

Suppose $X_{i} \sim \operatorname{ENDM}\left(\lambda_{i}, \mu_{i}, \beta_{i}\right)$ and it has the pdf as $f_{X_{i}}\left(x, \lambda_{i}, \mu_{i}, \beta_{i}\right)=\beta_{i} \exp \left\{-\lambda_{i}\left(x-\mu_{i}\right)^{2}\right\}$

Then consider the new random variable $X=\sum_{i=1}^{n} p_{i} X_{i}$ such that

$$
f_{X}(x, \lambda, \mu, \beta)=\sum_{i=1}^{n} p_{i} f_{X_{i}}\left(x, \lambda_{i}, \mu_{i}, \beta_{i}\right)=\sum_{i=1}^{n} p_{i} \beta_{i} \exp \left\{-\lambda_{i}\left(x-\mu_{i}\right)^{2}\right\}
$$


where $0 \leq p_{i} \leq 1, i=1, \ldots, n$ for $\sum_{i=1}^{n} p_{i}=1$ and $\left(\mu_{i}, \lambda_{i}, \sigma_{i}\right)$ represent the parameters of mixture model $\operatorname{ENDM}(3)$ of the $i^{\text {th }}$ component [4]. For $n=2$ suppose $X=p X_{1}+(1-p) X_{2}$ is a mixture of $X_{1}$ and $X_{2}$, the pdf of $X$ is

$f_{X}(x, \lambda, \mu, \beta)=p \beta_{1} \exp \left\{-\lambda_{1}\left(x-\mu_{1}\right)^{2}\right\}+(1-p) \beta_{2}$

$\exp \left\{-\lambda_{2}\left(x-\mu_{2}\right)^{2}\right\}$

Technically, the uncertainty in the net benefit function must be a normally distributed function for the so-called unit normal loss integral formula to be valid.

There are sets of original data that can be adequately described by a phenomena which are normally distributed. Reexamining sources of original data, we could not find any samples fitting the normal distribution that did not fit the log or multiplicative of models which are normally distributed equally well, or better of course, transformed data and other quantities derived from sources of the data often show a normally distributed.

Because of its edibility and several other interesting properties. Due to these properties, considerable results have been concluded by aspects of this new mixture of type of distributions. Earlier work on index focused upon relatively simple proposed data collection data on every uncertain model parameter in one single data collection with a single specified the case of sample data size and was valid in relatively small number contexts. The data used in this study were obtained from the literature.

The assumption that the normal mixture model should be more accurate than n-means is not necessarily true. There's no rule that says that it should always be better, particularly with something so subjective as image classification in Figure 4.

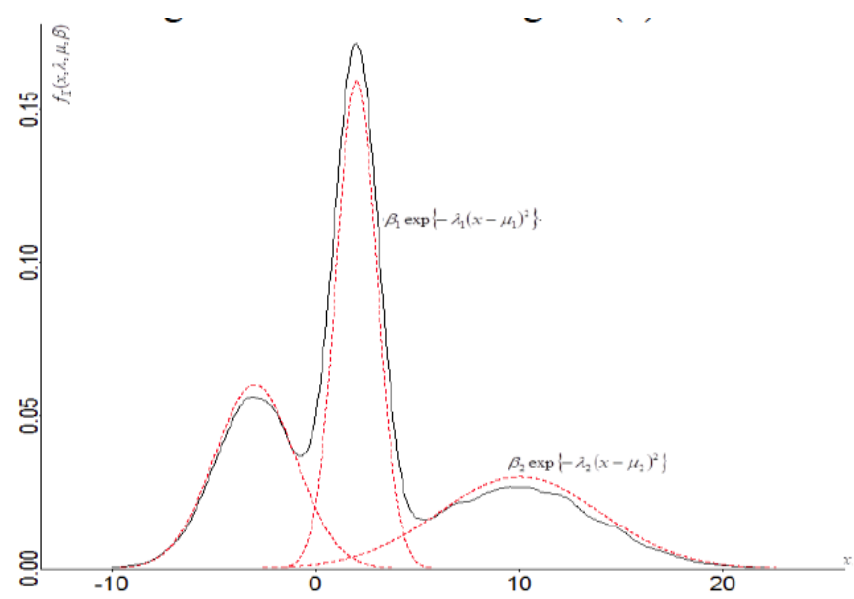

Figure 4: Graph of approximation of the mixture density.
Notice the distribution so that it looked good; it's longer a true probability density function. In this case, the scaling is actually the max value of the probability density function and then measure the weight by the mixture proportion. As to the speckle position.

However, while problems linked with mixture model distributions relate to deriving the results of different properties of the overall population from those of the sub-populations. I just used the data cursor, given only observations on the pooled population, without subpopulation identity information.

The combined two gaussian normal distributions with the same mean, but the resulting distribution is not normal, as can be seen by comparison to the superimposed normally curve.

The age distribution for children known to the with data in Table 1 looks normally distributed with an average age of 10.509 years compared to 8.2 years for children where the age profile does not have a gaussian normal distribution. The frequency distribution chart (See Figure 4) shows that there are more children known to the local authority within almost all of this range of ages apart from in children aged 0,1 and 2 . The mean age for all children in this study is 10.2 years. The prevalence of $(0-5)$ year olds is $2.2 \%$, which increases to $5.8 \%$ of $(5-10)$ year olds and $6.8 \%$ of (15$20)$ year olds. Confidence intervals suggest that there the prevalence of (0-5) year olds is significantly lower than the prevalence in children and (5-10) and (10-15).

\section{APPLICATIONS AND CONCLUSIONS}

The hospital is a statistically significant predictor of the costs and for this the cost function is useful for estimating the cost of specific type of patient with different characteristics and treatment conditions. In domain of statistics the sensitivity of analysis of the studied references showed many special and basic applications of some models derived from the general model of the extended normal distribution.

The variance is a measure of the variability of values around the mean and is meant to be used with values that have normal distribution with follow a normal curve.

Given that most cost-effectiveness models of interventions and most proposed research studies focus on more complex situations than this, a generalized method for calculating partial index is required. A method applies (using unit normal loss 
Table 1: Data for Medical Review in School for Ten Years

\begin{tabular}{|c|c|c|c|c|c|}
\hline Age Group & Number & Rate on (\%) & Number on register (\%) & Prevalence & 95\% Confidence level \\
\hline \hline$(0-5)$ & 2452 & 10.884 & $391(12.99 \%)$ & $13.509 \%$ & $0.8-3.7$ \\
\hline$(5-10)$ & 3443 & 15.223 & $913(30.22 \%)$ & $21.880 \%$ & $4.3-7.3$ \\
\hline$(10-15)$ & 2955 & 13.107 & $1034(34.22 \%)$ & $19.394 \%$ & $5.3-8.3$ \\
\hline$(15-20)$ & 2453 & 10.785 & $661(21.91 \%)$ & $17.209 \%$ & $3.0-6.2$ \\
\hline Unknown age(0-20) & 11303 & 50.11 & $22(0.71 \%)$ & $17.809 \%$ & \\
\hline
\end{tabular}

integral formula to calculate for sample size $n$ ), if the net benefit function is normally distributed, and if the proposed sample exercise measures all the parameters of the model.

The modelling of the results data in hospital has strong affinities with the represent model of data cost, as both have normally models with not free health care costs. The statistical assumptions and represented models were examined for the normally distributed, influential observations, multicollinearity, homoscedasticity and outliers [6].

The patients under the civil servant medical benefit scheme incur a higher cost than those under the universal health coverage scheme. This might be an effect of the health financing methods. Stepwise normal distribution analysis with two parameters [6] was employed to analyze the correlation between the cost (dependent variable) and potential predictor variables (independent variables).

Mixtures of gaussian normal distributions models, independent variables with a probability value of Fisher statistics $<0.05$ on the analysis were entered, and those with $>0.10$ were removed.

The result of statistic assumptions and models were studied for the following results: the gaussian normal distribution, influential observations, homoscedasticity and outliers. In the forward stepwise regression model, independent variables with a probability value of $F$ statistics $<0.05$ on the analysis were entered, and those with $>0.10$ were removed.

The statistical assumptions and models were examined for the following: normal distribution, homoscedasticity, multicollinearity, influential observations, and outliers [6]. To analyze robustness of the results due to sample data, univariate sensitivity analysis was employed [16]. The costs as outcome data represented by variables were transformed into a natural logarithm variable form to meet the assumption of gaussian normal distribution represented the case study [12]. Variation of the types of chelation and blood drugs was included in the results. Simulated scenarios were the nucleic acid tested of the type of the blood use instead of the filtered types of blood, and the oral form of iron chelation medicine use instead of the injection form.

\section{REFERENCES}

[1] Mohammad A, Golam Kibria BM, Mohammad S. Normal and Student's t Distributions and Their Applications. Atlantis Studies in Probability and Statistics, Series Editor: C.P.T Sokos 2014.

[2] Lyon A. Why are Normal Distributions Normal? British Journal for the Philosophy of Science 2014; 65(3): 621-649. https://doi.org/10.1093/bjps/axs046

[3] Lahcene B. On Recent Modifications of Extended Weibul Families Distributions and Its Applications Asian Journal Of Fuzzy And Applied Mathematics 2018; 6(1): 1-12.

[4] Lahcene B. On Recent Modifications of Extended Rayleigh Distribution and its Applications. JP Journal of Fundamental and Applied Statistics 2018; 12(1): 1-13.

[5] Box George EP, Tiao C. Bayesian Inference in Statistical Analysis. New York: Wiley 1992.

[6] Cohen J, Cohen P, West SG, Aiken LS. Applied Multiple Regression/ Correlation Analysis for the Behavioral Sciences. Third edition. New Jersey: Lawrence Erlbaum Associates, Inc. 2003.

[7] David FN. Games, Gods, and Gambling. New York, Hafner Pub. Co. 1962.

[8] Feller W. An introduction to probability theory and its Applications. Third Edition, New York: Wiley 1971; 2.

[9] Francis G. F.R.S Results Derived From the Natality Table of Körösi by Employing the Method of Contours or Isogens. Proceedings Royal Society of London 1894; 18(331): 18-23.

[10] Gravesande GJ. Demonstration Mathematique de la Direction de da Providence Divine. Oeuvres 1774; 2: 221 236.

[11] Hald A. A History of Probability and Statistics and Their Applications before 1750. New York, John Wiley 1990. https://doi.org/10.1002/0471725161

[12] Heyse JF, Cook JR, Carides GW. Statistical Considerations in Analysing Health Care Resource Utilization and Cost Data. In Economic Evaluation in Health Care - Merging Theory with Practice, Edited by Drummond M, McGuire A. New York: Oxford Univers ity Press 2001.

[13] Laplace, Pierre-Simon de. Memoire sur la Probabilite des Causes par les Evenements. Mémoires de l'Académie royale des Sciences de Paris (Savants étrangers), tome(6): pp 621656. Translated by Stephen M. Stigler in Statistical Science 1774; 1(3): 1986 
[14] Laplace PS. Memoire sur les Approximations des Formules qui sont Fonctions de tres Grand Nombres et sur leur Applications aux Probabilites. M'emoires de l'Academie des sciences de Paris: 1809; pp. 353-415 and 559-565.

[15] Lukas E. A Characterization of the Normal Dstribution. Annals of Mathematical Statistics 1942; 13: 91-93. https://doi.org/10.1214/aoms/1177731647

[16] Manning WG, Fryback DG, Weinstein MC. Reflecting Uncertainty in Cost-Effectiveness Analysis(In Costeffectiveness in health and medicine). Edited by Gold MR, Sigel JE, Russell LB, Weinstein MC. Oxford: Oxford University Press 1996.

[17] Jagdish PK, Campbell RB. Handbook of the Normal Distribution", Second Edition, CRC Press 1996.

[18] Pearson ES, Kendall M. Studies in the History of Statistics and Probability. London, Griffin 1970; 1: 185-189.
[19] Karl P. Notes on the History of Correlation. Biometrika 1920; 13(1): 25-45.

https://doi.org/10.1093/biomet/13.1.25

[20] Pierce RG, Bozic KJ, Hall BL, Breivis J. Health Care Technology Assessment: Implications for Modern Medical Practice 2007.

[21] Part I. Understanding Technology Adoption and Analyses, Am J Orthop 36(1): 11-14.

[22] Rorty R, Schneewind JB. The Taming of Chance. Edited By Quentin Skinner (General Editor), Lorraine Daston, Wolf Lepenies, University Press 2002.

[23] Stahl S. The Evolution of the Normal Distribution. Mathematics Magazine 2006; 79(2): 96-113. https://doi.org/10.1080/0025570X.2006.11953386 